International Journal of Mathematics and Systems Science (2018) Volume 1 doi:10.24294/ijmss.v1i3.546

\title{
A $\lambda$-c- genetic Algorithm for Integrals with Fuzzy Measure
}

\section{Gholam Hassan Shirdel}

Department of Mathematics, Faculty of Science, University of Qom, Qom, Iran, g.h.shirdel@qom.ac.ir shirde181math@gmail.com

\section{ABSTRACT}

In this paper, beginning we define a fuzzy Parametric measure, with having values of a weight function on $\mathrm{n}$ points. Afterwards, we obtain one equation by use from properties of fuzzy measure that with solving equation, we define parameters of fuzzy measure. For solving equation, we design a genetic algorithm and hereby we provide the facility of solving integrals.

Keywords: Fuzzy Measure; Fuzzy Integral; Genetic Algorithm

\section{Introduction}

In some cases, from a weight function is used for ranking, comparison and selection ${ }^{[3,6,9,12,14,18,20]}$. Ratings, comparison and selection can be done in many different ways $^{[12,14,16-20]}$. Since data is fuzzy in many systems that in them should be done process ranking, comparison and selection, therefore it is necessary that we design types of methods which with implementation them on fuzzy data, be done a good selection [3,6,9]. One of these methods is construct a fuzzy measure and fuzzy integral. In this paper, beginning we define a parametric-fuzzy measure, afterwards we solve a type of fuzzy integral by use from above fuzzy measure ${ }^{[1,2,5,10,11,13]}$. We need to amount of parameter of fuzzy measure for solving above fuzzy integrals. For find the amount of above parameter, we obtain one nonlinear equation with two unknown by use from properties of fuzzy measure. At the end, we design a genetic algorithm for solving the above nonlinear equation and by implementation it, obtain suitable approximation solutions.

\section{Basic Concepts}

This section introduces of the required methodologies.

\subsection{Fuzzy measure}

Sugeno defined fuzzy measure ${ }^{[1,8,11,19]}$. This concept is widely in various fields, especially in the field of collecting and summarizing information.

Definition 1. ${ }^{[7]}$ Assume that $X=\left\{x_{1}, X_{2}, \ldots, x_{n}\right\}$ and $P(X)$ is the power set of $X$, the set function $g: P(X)$ $\longrightarrow[0,1]$ is called a fuzzy measure, which is non-additive and preserves the following properties

$$
\begin{aligned}
& \text { 1. } g(\varnothing)=0 \text {, } \\
& \text { 2. } g(X)=1 \text {, } \\
& \text { 3. if } A, B \in P(X) \text { and } A \subset B \text { then } g(A) \leq g(B)
\end{aligned}
$$
(monotonicity),

4. in $P(X)$, if $A_{1} \subset A_{2} \subset A_{3} \subset A_{4} \subset \ldots$ and $\bigcup_{i=1}^{\infty} A_{i} \in P(X)$ then $\lim _{i} g\left(A_{i}\right)=g\left(\bigcup_{i=1}^{\infty} A_{i}\right)$ (continuity from below),

5. in $P(X)$, if $A_{1} \supset A_{2} \supset A_{3} \supset A_{4} \supset \ldots$ and $\bigcap_{i=1}^{\infty} A_{i} \in P(X) \quad, \quad$ then $\quad \lim _{i \rightarrow \infty} g\left(A_{i}\right)=g\left(\bigcap_{i=1}^{\infty} A_{i}\right)$ (continuity from below).

Now we can give the definition of fuzzy parametricmeasure as following.

Defination 2. ${ }^{[7]}$ The set function $g_{\lambda}: P(X) \longrightarrow[0,1]$ is called a fuzzy $\lambda$-measure if and only if there be a parameter $\lambda$ such that $\lambda \in\left(\frac{-1}{\operatorname{supg}}, \infty\right)$ which $\operatorname{supg}=$ $\sup _{A \in(X)} g(A)$ and $\mathrm{A} \in \mathrm{P}(\mathrm{X})$

$$
g_{\lambda}(A \cup B)=g_{\lambda}(A)+g_{\lambda}(B)+\lambda g_{\lambda}(A) g_{\lambda}(B)
$$

that $A, B \in P(X), A \cap B=\emptyset$. This measure is $\lambda$ -additive.

\subsection{Fuzzy Integrals}

Assume that $X=\left\{x_{1}, x_{2}, \ldots, x_{n}\right\}$ and $g$ is a fuzzy measure on $X$. Integral of a function $h: P(X) \longrightarrow[0,1]$ with respect to $\mathrm{g}$ is defined by $\int \mathrm{fdg}:=\sum_{\mathrm{i}=1}^{\mathrm{n}}\left(\mathrm{h}\left(\mathrm{x}_{\mathrm{i}}\right)-\right.$ $\left.\mathrm{h}\left(\mathrm{x}_{\mathrm{i}+1}\right)\right) \mathrm{g}\left(\mathrm{H}_{\mathrm{i}}\right) \quad$ where $\quad 0 \leq \mathrm{h}\left(\mathrm{x}_{\mathrm{n}}\right) \leq \mathrm{h}\left(\mathrm{x}_{\mathrm{n}-1}\right) \leq \ldots \leq$ $h\left(x_{1}\right) \leq 1, h\left(x_{n+1}\right)=0$ and $H_{i}=\left\{x_{1}, x_{2}, \ldots, x_{i}\right\}$.

Copyright (C) 2018 Gholam Hassan Shirde

doi: 10.24294/ijmss.v1i3.546

EnPress Publisher LLC.This work is licensed under the Creative Commons Attribution-NonCommercial 4.0 International License (CC BY-NC 4.0). http://creativecommons.org/licenses/ by/4.0/ 
In literature, the fuzzy integral defined by $\int \mathrm{h} d g$ is called "Choquet integral". The basic concept can be illustrated in Figure 1 ${ }^{[13]}$.

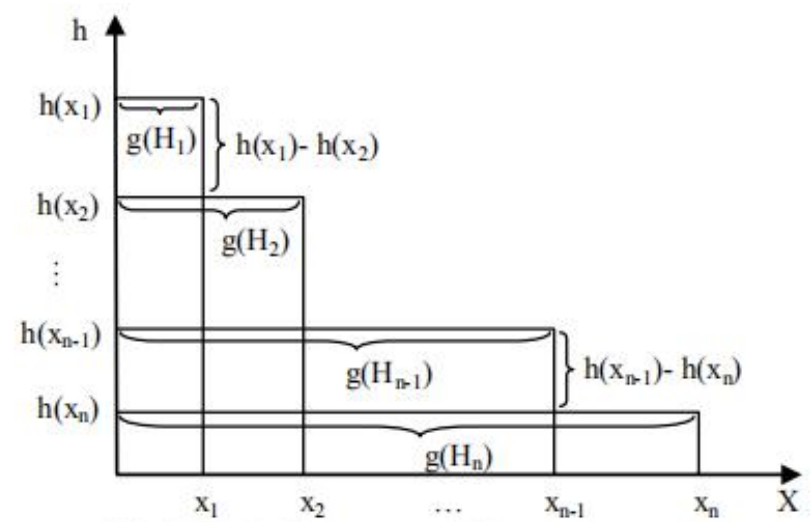

Figure 1; The basic concept of Choquet integral.

Lemma 1. Suppose $X=\left\{x_{1}, x_{2}, \ldots, x_{n}\right\}$, therefore for any fuzzy measure $\mathrm{g}$ on $\mathrm{X}$ and for any function $\mathrm{h}: \mathrm{X}$ $\longrightarrow[0,1]$, it is verified that $\int h d g=\sum_{i=1}^{n} h_{i} d g_{i}$.

Proof. According to the definition of the Choquet integral, we have

$$
\int f d g:=\sum_{i=1}^{n}\left(h\left(x_{i}\right)-h\left(x_{i+1}\right)\right) g\left(H_{i}\right) .
$$

Now since $\mathrm{h}\left(\mathrm{x}_{\mathrm{n}+1}\right)=0, \mathrm{~g}_{0}=\mathrm{g}(\varnothing)=0$ and $\mathrm{g}(\mathrm{X})=\mathrm{g}\left(\mathrm{H}_{\mathrm{n}}\right)=1$, we have

$$
\begin{gathered}
\sum_{i=1}^{n}\left(h_{i}-h_{i+1}\right) g_{i}=\sum_{i=1}^{n} h_{i} g_{i}-\sum_{i=1}^{n} h_{i+1} g_{i} \\
=\sum_{i=1}^{n} h_{i} g_{i}-\sum_{i=2}^{n+1} h_{i} g_{i-1} \\
=h_{1} g_{1}+\sum_{i=2}^{n} h_{i} g_{i}-\sum_{i=2}^{n} h_{i} g_{i-1} \\
-h_{n+1} g_{1} \\
=h_{1} g_{1}-h_{1} g_{0}+\sum_{i=2}^{n} h_{i}\left(g_{i}-g_{i-1}\right)=\sum_{i=1}^{n} h_{i}\left(g_{i}-g_{i-1}\right) \\
=\sum_{i=1}^{n} h_{i} d g_{i} . \square
\end{gathered}
$$

\section{Calculating the fuzzy integral}

We obtain parameter of fuzzy measure for this purpose, for any $i=1,2, \ldots, n$ we set

$$
g_{i}=g\left(\left\{x_{i}\right\}\right)=c w_{i}
$$

which $c$ is a constant real number and $w_{i}$ is amount of a weight function on $x_{i}^{[2,5,13]}$.

Now according to (1), (2) and $g(X)=1$, we have

$$
\begin{gathered}
c \sum_{\alpha_{1}=1}^{\mathrm{n}} \mathrm{w}_{\alpha_{1}}+\mathrm{c}^{2} \lambda \sum_{\substack{\alpha_{1}=1, \alpha_{2}=1 \\
\alpha_{1} \neq \alpha_{2}, \alpha_{1}<\alpha_{2}}}^{\mathrm{n}} \mathrm{w}_{\alpha_{1}} \mathrm{w}_{\alpha_{2}} \\
+\mathrm{c}^{3} \lambda^{2} \sum_{\substack{\alpha_{1}=1, \alpha_{2}=1, \alpha_{3}=1 \\
\alpha_{1} \neq \alpha_{2} \neq \alpha_{3}, \alpha_{1}<\alpha_{2}<\alpha_{3}}} \mathrm{w}_{\alpha_{1}} \mathrm{w}_{\alpha_{2}} \mathrm{w}_{\alpha_{3}} \\
+\ldots+\mathrm{c}^{\mathrm{n}} \lambda^{\mathrm{n}-1} \prod_{\alpha_{\mathrm{i}}=1}^{\mathrm{n}} \mathrm{w}_{\alpha_{\mathrm{i}}}-1=0
\end{gathered}
$$

Equality (3) is a nonlinear equation with two unknown $\lambda$, $c$ and can be re-written as following

$$
\mathrm{F}(\mathrm{c}, \lambda)=\sum_{\mathrm{i}=1}^{\mathrm{n}} \mathrm{a}_{\mathrm{i}} \mathrm{c}^{\mathrm{i}} \lambda^{\mathrm{i}-1}-1=0
$$

Which

$\mathrm{a}_{1}=\sum_{\alpha_{1}=1}^{\mathrm{n}} \mathrm{w}_{\alpha_{1}}, \quad \mathrm{a}_{2}=\sum_{\substack{\alpha_{1}=1, \alpha_{2}=1 \\ \alpha_{1} \neq \alpha_{2}, \alpha_{1}<\alpha_{2}}}^{\mathrm{n}} \quad \mathrm{w}_{\alpha_{1}} \mathrm{w}_{\alpha_{2}}, \quad \mathrm{a}_{3}=$ $\sum_{\substack{\alpha_{1}=1, \alpha_{2}=1, \alpha_{3}=1 \\ \alpha_{1} \neq \alpha_{2} \neq \alpha_{3}, \alpha_{1}<\alpha_{2}<\alpha_{3}}}^{\mathrm{n}} \quad \mathrm{w}_{\alpha_{1}} \mathrm{w}_{\alpha_{2}} \mathrm{w}_{\alpha_{3}}$ $\mathrm{a}_{\mathrm{n}}=\prod_{\alpha_{\mathrm{i}}=1}^{\mathrm{n}} \mathrm{w}_{\alpha_{\mathrm{i}}}$.

Now we design a $\lambda$-c-genetic algorithm for solving equation (4). By solving equation (4), we obtain approximation amount of $c, \lambda$ with the accuracy of pre-defined.

\subsection{The $\lambda$-c-genetic algorithm}

begin

Best_c $=0$;

Best_lamda $=0$;

alfa $=$ Producealfa $(n, w) ;$

for $\mathrm{i}=1: \mathrm{k}$

$$
c(i)=\frac{i}{b}
$$

end

$t=\frac{k}{2}$

For $\mathrm{i}=1: \mathrm{t}$

$$
\begin{aligned}
& \operatorname{lamda}(\mathrm{i})=\frac{-i}{t} \\
& \operatorname{lamda}(\mathrm{i}+\mathrm{t})=\frac{i}{t} \\
& \operatorname{lamda}(\mathrm{i}+2 * \mathrm{t})=i^{\wedge} 2+1
\end{aligned}
$$

end

Term $=0$;

$\mathrm{nk}=3 * \mathrm{k}$;

Gen $=1$;

while Term $\sim=1 \& \&$ Gen $<=100$

for $\mathrm{i}=1: \mathrm{k}$

$$
\begin{aligned}
& \mathrm{X}=\operatorname{rand} \mathrm{i}(\mathrm{k}, 1,2,) \\
& \mathrm{l}=\mathrm{X}(1) \\
& \mathrm{f}=\mathrm{X}(2) \\
& c(k+i)=(c(l)+c(f)) / 2 \\
& c(2 * k+i)=c(l) * c(f) \\
& \operatorname{lamd} a(k+i)=(\operatorname{lamda}(l)+\operatorname{lamd} a(f)) / 2
\end{aligned}
$$




$$
\operatorname{lamda}(2 * k+i)=\operatorname{abs}(c(l) * c(f)) ;
$$

End
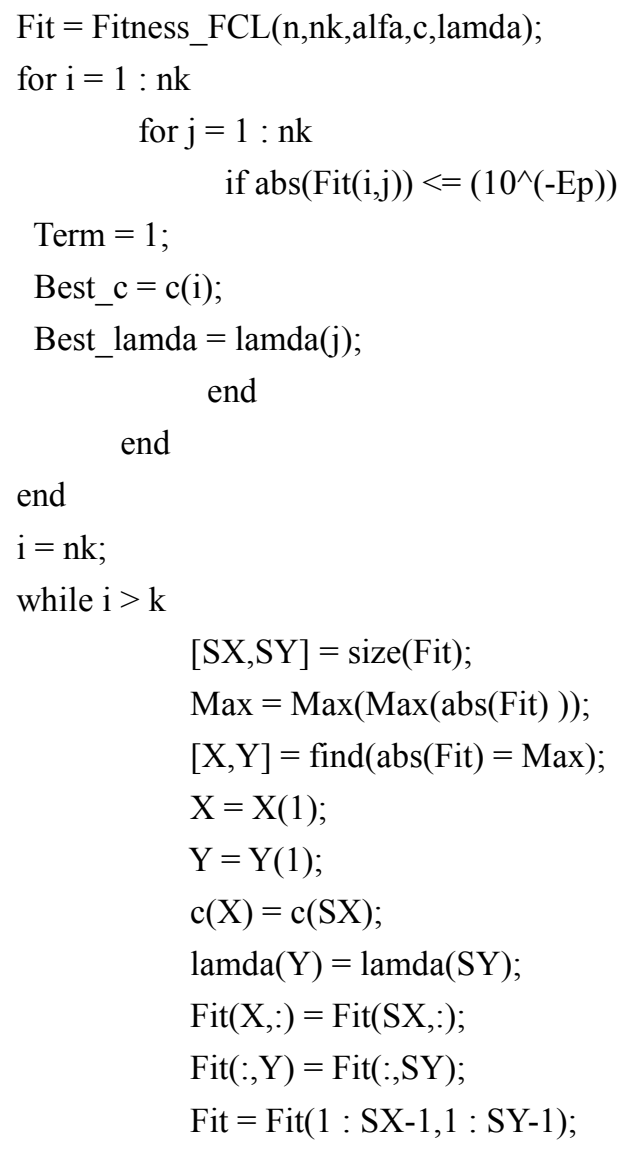

end

Gen $=$ Gen +1 ;

function Fitness $=$ Fitness_FCL(n,k,alfa,lamda)

for $\mathrm{X}=1: \mathrm{k}$

for $\mathrm{Y}=1: \mathrm{k}$

Fitness $(\mathrm{X}, \mathrm{Y})=0$;

for $\mathrm{i}=1: \mathrm{n}$

Fitness $(\mathrm{X}, \mathrm{Y})=$ Fitness $(\mathrm{X}, \mathrm{Y})+$

$\operatorname{alfa}^{*}\left(\mathrm{c}(\mathrm{x})^{\wedge} \mathrm{i}\right)^{*}\left(\operatorname{lamda}(\mathrm{Y})^{\wedge}(\mathrm{i}-1)\right)$;

end

Fitness $(X, Y)=$ Fitness $(X, Y)-1$; end

end

function alfa $=$ Producealfa $(\mathrm{n}, \mathrm{w})$

alfa $(1: \mathrm{n})=0$;

for $\mathrm{X}=1: \mathrm{n}$

start_index $(1: \mathrm{X})=1: \mathrm{X}$;

stop_index $(1: \mathrm{X})=\mathrm{n}-\mathrm{X}+1: \mathrm{n}$;

while start_index $(1)<=$ stop_index(1)

$\mathrm{c}=0$;

$\mathrm{P}=1$; for $\mathrm{z}=1: \mathrm{X}$

$$
\mathrm{P}=\mathrm{P} * \mathrm{~W}\left(\operatorname{start} \_ \text {index }(\mathrm{z})\right) \text {; }
$$

end

$\operatorname{alfa}(X)=\operatorname{alfa}(X)+P$;

$\mathrm{z}=1$;

while $\mathrm{z}<=\mathrm{X} \& \& \mathrm{c}=0$

$$
\text { if start_index }(\mathrm{z})=\text { stop_index }(\mathrm{z})
$$

if $\mathrm{z}=1 \& \&$ isempty(find(start_index $=$ stop_index) $=0)$ )

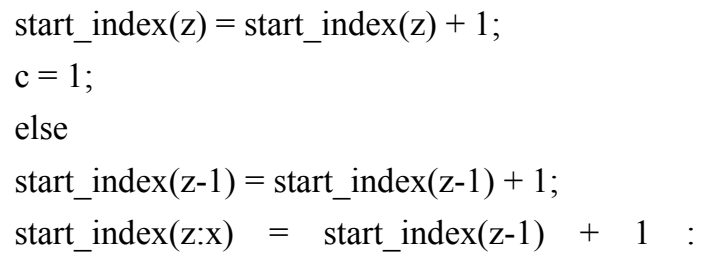

else

start_index $(z-1)=$ start_index $(z-1)+1$;

start_index $(\mathrm{z}: \mathrm{x})=$ start_index $(\mathrm{z}-1)+1$ :

start_index $(\mathrm{z}-1)+\mathrm{X}-\mathrm{z}+1$;

$\mathrm{c}=1$

end

end

$\mathrm{z}=\mathrm{z}+1$

end

$\mathrm{z}=\mathrm{z}-1$

if $\mathrm{z}=\mathrm{X} \& \&$ start_index $(\mathrm{z})<\operatorname{stop}$ index $(\mathrm{z})$

$\& \& \mathrm{c}=0$

$$
\text { start_index }(\mathrm{z})=\text { start_index }(\mathrm{z})+1 ;
$$

end

end

end

\subsection{Example}

Consider $X=\{1,2,3\}, \quad g_{\lambda}: P(X) \longrightarrow[0,1], h: X$ $\longrightarrow[0,1]$, with $h(x)=e^{-x}$ and $w_{1}=w_{2}=w_{3}=\frac{1}{3}$. So, we have $w_{1}+w_{2}+w_{3}=1, \quad w_{1} w_{2}+w_{1} w_{3}+$ $w_{2} w_{3}=\frac{1}{3}, w_{1} w_{2} w_{3}=\frac{1}{27}, c+\frac{1}{3} c^{2} \lambda+\frac{1}{27} c^{3} \lambda^{2}-1=0$.

By implementation $\lambda$-c-genetic algorithm, we obtain that $c=\frac{1}{2}$ and $\lambda=5$. Thus we have $g_{1}=\frac{1}{6}, g_{2}=\frac{1}{6}$, $g_{3}=\frac{1}{6}, g\left\{x_{1}, x_{2}\right\}=\frac{17}{36} \quad$ and $g\left\{x_{1}, x_{2}, x_{3}\right\}=\frac{223}{216} \quad$ which imply that

$$
\int h d g:=\sum_{i=1}^{3}\left(h\left(x_{i}\right)-h\left(x_{i+1}\right)\right) g\left(H_{i}\right)=0.128 . \square
$$

\section{References}

1. A. Basile, Fuzzy Sets and Systems 21, 243-247 (1987).

2. M. T. Chu, Z. Shyu, J. and G. H. Tzeng, IEEE Transactions on Engineering Management 54 (2), 237-339 (2007).

3. C. M. Feng, P. G. Wu and K. C. Chia, European Journal Operational Research 192, 451-464 (2009). 
4. D. E. Goldberg, Addison-Wesley, Reading, MA, 1989.

5. L. Galand, P. Perny, O. Spanjaard, European Journal of Operational Research 204 (2), 303-315 (2010)

6. M. Grabisch, European Journal of Operational Research 89 (3), 445-456 (1996).

7. M. Grabisch, Fuzzy measures and integrals, Springer Verlag (2000).

8. M. Grabisch, T. Murofushi and M. Sugeno, Fuzzy Sets and Systems 92, 167-189 (1997).

9. M. Grabisch, Fuzzy Sets and Systems 69, 279-298 (1995).

10. Y. C. Hu, Neurocomputing 72, 331-340 (2008).

11. K. Leszczynski, P. Penczek and W. Grochuliski, Fuzzy Sets and Systems 15, 147-158 (1985).

12. D. Liginlal and T. T. Ow, Fuzzy Sets and Systems 157, 3040-3054 (2006).

13. T. Murofushi and M. Sugeno, Fuzzy Sets and systems 29, 201-227 (1989).

14. Y. Narukawa and V. Torra, Information Sciences 177, 4686-4695 (2007).

15. Y. Narukawa, T. Murofushi and M. Sugeno, Fuzzy Sets and systems 112, 177-186 (2000).

16. T. Onisawa, M. Sugeno, M. Y. Nishiwaki, H. Kawai and Y. Harima, Fuzzy Sets and Systems 20, 259-289 (1986).

17. M. Sugeno, Theory of fuzzy integrals and applications, Ph.D. Dissertation, Tokyo Institute of Technology, 1974.

18. G. H. Tzeng, Y. P. Q. Yang, C. T. lin and C. B. Chen, Information Sciences 169, 409-426 (2005).

19. Z. Wang and G. J. Klir, Fuzzy measure theory, Plenum Press, New York, 1992.

20. S. T. Wierzchon, Fuzzy Sets and Systems 9, 69-78(1983) 